\title{
Anterior Lumbar Interbody Fusion: Two-Year Results with a Modular Interbody Device
}

\author{
Keith Lynn Jackson ${ }^{1}$, Chevas Yeoman ${ }^{2}$, Woosik M. Chung ${ }^{3}$, James L Chappuis ${ }^{4}$, Brett Freedman ${ }^{2}$ \\ ${ }^{1}$ Division of Orthopaedic Spinal Surgery, University of Pittsburgh Medical Center, Pittsburgh, PA, USA \\ ${ }^{2}$ Landstuhl Regional Medical Center, Landstuhl, Germany \\ ${ }^{3}$ Colorado Comprehensive Spine Institute, Englewood, CO, USA \\ ${ }^{4}$ Spine Center Atlanta, Atlanta, GA, USA
}

\begin{abstract}
Study Design: Retrospective case series.
Purpose: To present radiographic outcomes following anterior lumbar interbody fusion (ALIF) utilizing a modular interbody device. Overview of Literature: Though multiple anterior lumbar interbody techniques have proven successful in promoting bony fusion, postoperative subsidence remains a frequently reported phenomenon.

Methods: Forty-three consecutive patients underwent ALIF with $(n=30)$ or without $(n=11)$ supplemental instrumentation. Two patients underwent ALIF to treat failed posterior instrumented fusion. The primary outcome measure was presence of fusion as assessed by computed tomography. Secondary outcome measures were lordosis, intervertebral lordotic angle (ILA), disc height, subsidence, Bridwell fusion grade, technical complications and pain score. Interobserver reliability of radiographic outcome measures was calculated.

Results: Forty-three patients underwent ALIF of 73 motion segments. ILA and disc height increased over baseline, and this persisted through final follow-up $(p<0.01)$. Solid anterior interbody fusion was present in 71 of 73 motion segments $(97 \%)$. The amount of new bone formation in the interbody space increased over serial imaging. Subsidence $>4 \mathrm{~mm}$ occurred in $12 \%$ of patients. There were eight surgical complications (19\%): one major (reoperation for nonunion/progressive subsidence) and seven minor (five subsidence, two malposition).

Conclusions: The use of a modular interbody device for ALIF resulted in a high rate of radiographic fusion and a low rate of subsidence. The large endplate and modular design of the device may contribute to a low rate of subsidence as well as maintenance of ILA and Iordosis. Previously reported quantitative radiographic outcome measures were found to be more reliable than qualitative or categorical measures.
\end{abstract}

Keywords: Lumbar spine; Low back pain; Interbody cage

\section{Introduction}

When anterior lumbar interbody fusion (ALIF) was first introduced as a surgical technique, non-union and subsidence were frequently noted [1-3]. However ALIF performed with modern techniques employing interbody cages and bone graft substitutes has yielded higher fusion rates $(87 \%-100 \%)[4-8]$. Multiple investigators have also reported significant and durable improvements in validated patient outcome measures following ALIF $[4,9]$.

Received Nov 11,2013; Revised Nov 27, 2013; Accepted Dec 15, 2013

Corresponding author: Keith Lynn Jackson

Division of Orthopaedic Spinal Surgery, University of Pittsburgh Medical Center,

3471 Fifth Avenue, Kauffman Building Suite 1010, Pittsburgh, PA 15213, USA

Tel: +1-706-755-4937, Fax: +1-412-687-3724, E-mail: Lynn.jackson9@gmail.com 
Despite these results, subsidence has remained a problem with the use of both structural bone grafts and cage devices. In two studies examining postoperative subsidence Choi et al. [6] and Cheung et al. [5] noted at least $2 \mathrm{~mm}$ loss of intervertebral height in $77 \%$ of patients treated with a cage and $86 \%$ who received structural iliac crest bone graft. Potential sequelae of intervertebral height loss include foraminal stenosis, loss of sagittal alignment and fixed sagittal imbalance.

An ideal interbody spacer would resist axial compressive loads to provide early stability while transmitting appropriate compressive forces to the grafted material to encourage bony fusion. To limit subsidence, an implant should seek to overlay the periphery of the osseous endplate, which are the strongest areas of bone [10]. A wider endplate-implant interface also demonstrates higher loads to failure [11]. Therefore a device that provides contact with a larger area and engages the peripheral portions of the endplate may best reduce implant subsidence.

Modular design has been a common solution to the wide variation in anatomic size and shape between patients for a multitude of orthopaedic applications. While expandable modular devices have been utilized in spinal surgery for vertebral body replacement following corpectomy, the radiographic outcomes of a modular device utilized for ALIF have not been reported, to our knowledge. The purpose of this study is to evaluate radiographic outcomes and technical complications following ALIF using a modular interbody device.

\section{Materials and Methods}

This study is a retrospective case series of forty-three consecutive patients who underwent ALIF with a modular metallic intervertebral device (InFix, Zimmer, Warsaw, IN, USA) in seventy-three motio segments. A single surgeon (J.L.C.) completed all cases. The device is composed of titanium and consists of five components: two endplates, two vertical struts and an optional posterior endcap (for use in cases where the posterior annulus and longitudinal ligaments have been resected). While approved for treatment of single or 2-level lumbar degenerative disc disease in other countries (most commonly at the two most caudal segments), in the United States, this device is currently only approved for replacement of a resected or excised vertebral body.

All cases were performed through a left-sided para- median retroperitoneal approach with the assistance of an access surgeon. Upon completion of the exposure, a discectomy and standard endplate preparation were performed. The posterior annulus and posterior longitudinal ligament were left intact, except in the presence of preoperative lower extremity symptoms, in which case the compressive portion of the posterior annulus was resected. If the posterior annulus was removed or incompetent, the posterior end cap was utilized to prevent extravasation of the bone graft substitute into the epidural space. After preparation the disc space was trialed to the desired endplate coverage, height and lordotic angulation. The assembled implant was filled with a bone morphogenic- 2 protein-soaked collagen sponge (size medium or large, INFUSE, Medtronic, Memphis, TN, USA) and loaded on an insertion instrument. Diagnoses for patients undergoing ALIF included: spondylolisthesis $(\mathrm{n}=8$, six Meyerding grade 1 , two grade 2 ); pseudarthrosis ( $\mathrm{n}=3$, two posterior and one anterior); and discogenic syndrome $(\mathrm{n}=32)$.

Single level fusion was performed in twenty patients. Seventeen patients underwent 2-level fusion (One 2-level fusion used a different cage at one level. This level was excluded from analysis, but both levels did go onto solid fusion). Four patients underwent 3-level fusion while three individuals had fusion at 4 levels. At the time of ALIF, twenty-one patients underwent primary posterior spinal instrumentation with pedicle screw and rod construct, and two had existing posterior instrumentation from a previous failed fusion attempt. Eight patients received anterior instrumentation with plating. One patient received both an anterior plate and posterior instrumented fusion at the index procedure. Thus a total of thirty patients had supplemental fixation added at the time of the ALIF procedure. Eleven patients underwent stand-alone ALIF (nine single-level, two double-level), and two patients underwent ALIF following failed posterior spinal fusion.

Routine follow-up visits were scheduled at 6 weeks, 3-6 months, 1-2 years postoperatively and/or until radiographic fusion was observed on plain radiographs or computed tomography (CT). All imaging studies were evaluated by an independent fellowship-trained spine surgeon. Measured parameters included component position, migration, subsidence, endplate notching and intervertebral lordotic angle (ILA). Each operative level was radiographically assigned a modified Bridwell fusion grade (grade 1, fused with bridging bone present; 2, central bone growth and partial incorporation, but lucency 


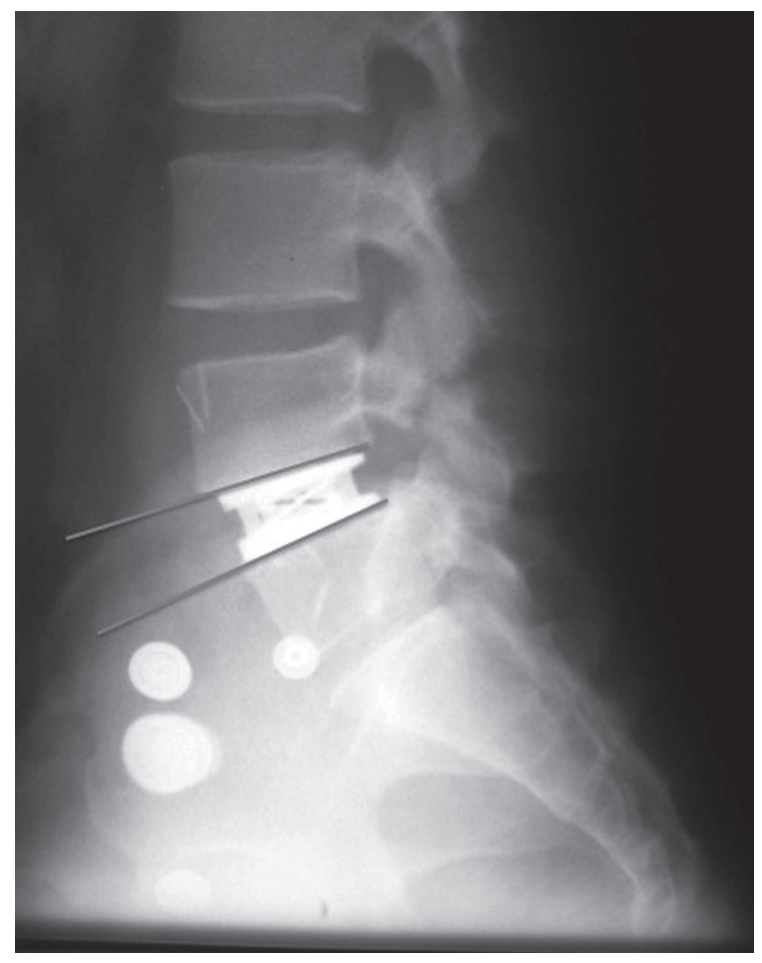

Fig. 1. Measurement of the intervertebral lordotic angle (ILA): ILA is the angle between lines connecting the anterior and posterior corners of the superior and inferior vertebral endplates.

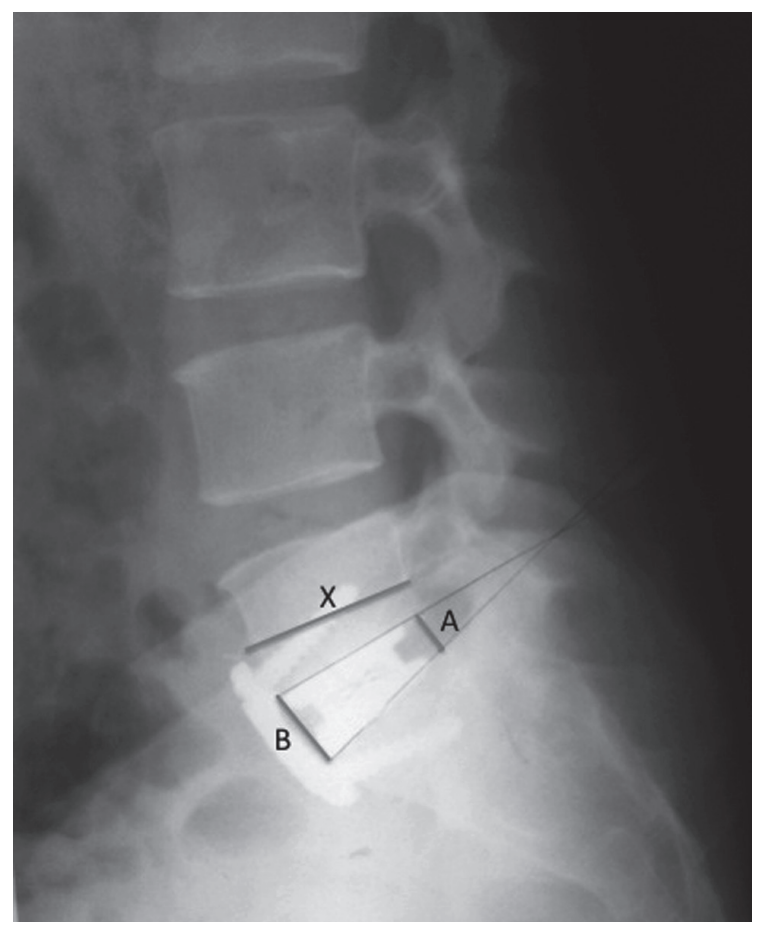

Fig. 2. Measurement of disc height: disc height is the average of the posterior vertical height $\mathbf{A}$ and the anterior vertical height $\mathbf{B}$. This value is then normalized to exclude the impact of magnification by multiplying by the quotient of the anterior-posterior diameter (horizontal line) of the current time-point by the preoperative anterior-posterior diameter. at top or bottom of cage; 3 , fusion absent with minimal bone growth; 4, definite nonunion, with no bone growth). ILA was defined as the angle formed by a line connecting the anterior-inferior and posterior-inferior corners of the superior vertebral body and a second line connecting the anterior-superior and posterior-superior corners of the inferior vertebral body on the lateral radiograph (Fig. 1). Subsidence was determined through the comparison of intervertebral disc heights over time as described by Cheung et al. $[5,6]$. Disc height is the average of two lines connecting the anterior and posterior corners of the superior and inferior vertebral bodies and accounts for magnification by measurement of the vertebral diameter at the inferior surface of the pedicle (Fig. 2). Subsidence was defined as decrease in disc height $\geq 4 \mathrm{~mm}(=2 \times$ measurement error) at any point in time compared to the initial postoperative disc height measurement. Fusion rate, number of bone bridges and percentage of disc space filled with bone were determined by CT (Fig. 3). Fusion

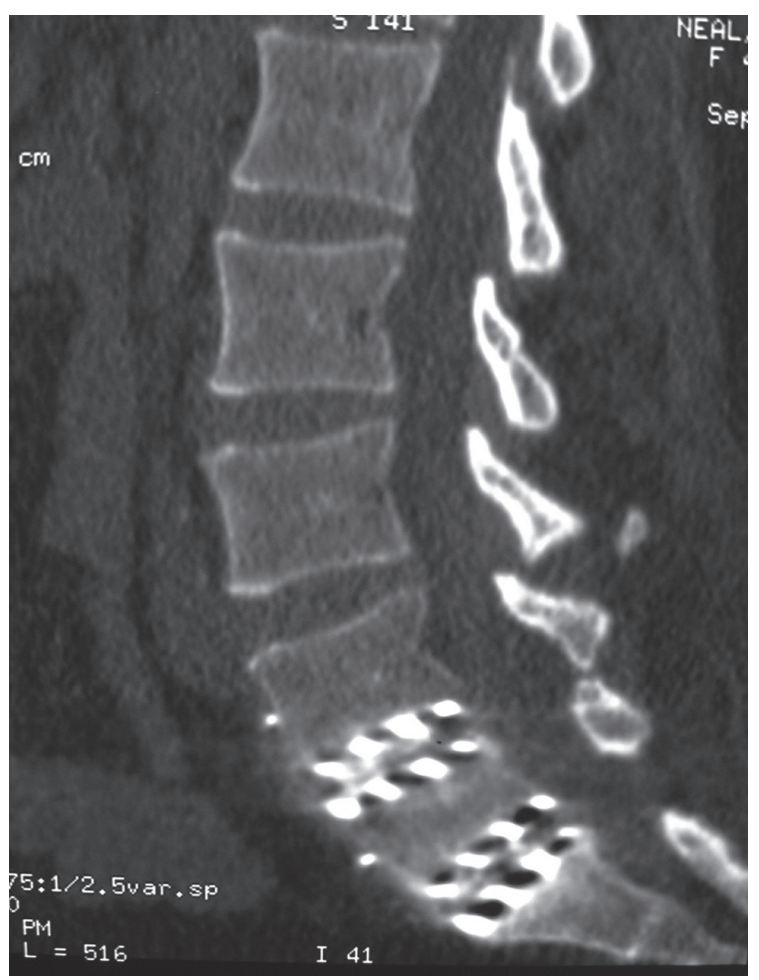

Fig. 3. Sagittal reconstruction at four months postoperative, from a patient with a two-level, stand-alone instrumented intervertebral lordotic angle, showing five bridges (note there is a propensity for focalized artifact [black spot] between fenestrations in the endplate component, but no lysis about the endplate-implant interface). This image also demonstrates $75 \%$ sagittal fill at $L 4 / 5$ and $100 \%$ sagittal fill and L5/1 with 5 bony bridges. 
was defined as a continuous bridge of bone between the superior and inferior vertebral endplates on at least two consecutive CT slices in either the sagittal or the coronal planes. In two cases in which postoperative CT scans were not performed, fusion status was determined by the Bridwell grade (grade 1). The number of bone bridges was quantified by counting the maximum number of continuous bony bridges (out of five possible) that connected the endplates on the sagittal and coronal images (Fig. 2). Percent fill was rounded to the nearest quartile. For eighteen cases (29 levels), repeat measurements were performed by a separate spine-fellowship trained observer to calculate interobserver reliability of the measures used in this study. Patients were asked the average back pain and the maximal back pain scores over the last four weeks at each clinic visit. This was reported on a 10-point pain scale, with 0 being no pain and 10 being the worst pain experienced.

Statistical analysis was carried out using STATA software. Strength of relationships between variables was calculated using linear regression $r^{2}$-values for correlation, while the coefficients and $t$-test values were used to assess significant change. Preoperative versus postoperative and first CT versus second CT values were compared using paired two tailed $t$-tests for significant differences. Interobserver reliability was measured for certain values that were obtained through repeated reads of radiographs and CTs. For the continuous measures of ILA and subsidence, an intraclass correlation coefficient (ICC) was obtained through one-way analysis of variance between the primary observer and independent repeats. Cohen's kappa statistic was calculated for the categorical and ordinal variables of the number of bony bridges, Bridwell scores and percent fill, as measured by two independent raters. Expected agreement percentage on chance alone and actual agreement percentage were also reported for kappa statistics.

\section{Results}

During a 22-month period (May 2005 to March 2007), 43 patients underwent ALIF involving 73 motion segments. Follow-up was available for all patients (mean followup, 584 days; range, 120-1,733 days; standard deviation $[S D]=336.4$ ). For $79 \%$ of patients, final follow-up was $\geq 1$ year from the date of surgery. All nine patients with $<12$ months follow-up had solid fusion, the primary outcome measure, on their final imaging studies (range, 120-314 days). Mean age was 40 years (range, 21-67 year; $\mathrm{SD}=10.6$ ), with $70 \%$ being male. Twenty-four percent were obese, with a mean body mass index of 28 (range, $20-40 ; \mathrm{SD}=4.1$ ). Thirty-five percent of the patients were smokers; and 56\% were involved in a worker's compensation claim.

Mean estimated blood loss (EBL) was $400 \mathrm{~mL}$ (range, 50-1500 mL, $\mathrm{SD}=385.4)$, and mean total operative time was 5.0 hours (range, 1.5-9.0 hours; $\mathrm{SD}=2.2$ ). EBL increased with increasing number of levels decompressed $(p<0.001)$, at an estimated rate of $363 \mathrm{~mL}$ for every level. Time in the operating room increased with increasing number of levels decompressed $(p<0.001)$, at an estimated rate of 1.5 hours additional for every level. Mean preoperative VAS average pain score was 7.0 (range, 2.5-9.5; $\mathrm{SD}=1.4$ ) with a mean VAS maximum pain score of 8.4 (range, $5.0-10 ; \mathrm{SD}=1.3$ ). The VAS average pain score decreased at final follow-up by a mean of 1.9 points $(p<0.001)(\mathrm{SE}=0.3 ; 95 \%$ confidence interval $[\mathrm{CI}]=1.3,2.5)$ to 5.0 (range, $0.0-9.0 ; \mathrm{SD}=2.0$ ). The mean VAS maximum pain score decreased 2.0 points $(p<0.001)(\mathrm{SE}=0.3 ; 95 \%$ $\mathrm{CI}=1.4,2.6$ ) to 6.4 (range, $0.0-10 ; \mathrm{SD}=2.0$ ) by the time of final follow-up. Three patients (7\%) reported increased back pain at final follow-up, with a mean increase of 0.7 points (range, $0.5-1.0$ points; $S D=0.29$ ). No medical complications were detected within the limits of our data abstraction methodology.

CT scan to assess fusion status was performed in 41 of 43 patients postoperatively (mean $=128$ days [ $S D=123.6]$; range, 13-734 days) and revealed fusion in all levels. In two patients, postoperative CT scans were not obtained. In one case, progressive subsidence and obvious nonunion was noted on plain films of an L5-S1 ALIF with anterior plate supplemental fixation. The other case was a 3-level ALIF with supplemental pedicle screw instrumentation, who had Bridwell grade 1 fusion status at two levels and grade 2 at the third level with solid posterior fusion at final radiographic follow-up (392 postoperative days). Thus, the overall reported ALIF fusion rate for this study is 41 of 43 patients (95.3\%) or 71 of 73 levels (97\%). Solid fusion of all operated levels occurred in 20 of 21 patients with index ALIF and posterior instrumentation; 11 of 11 patients with stand-alone ALIF; and 8 of 9 patients with ALIF and anterior plating.

On the initial CT, the means for number of bony bridges and percent fill were 3.7 and $84.8 \%$ respectively for sagit- 
Table 1. Radiographic outcomes: ILA, height gain and average subsidence measures at all followup intervals

\begin{tabular}{|c|c|c|c|c|c|c|}
\hline \multirow{2}{*}{ X-ray measures } & \multicolumn{2}{|c|}{$\operatorname{ILA}\left({ }^{\circ}\right)$} & \multicolumn{2}{|c|}{ Height gain (mm) } & \multicolumn{2}{|c|}{ Subsidence (mm) } \\
\hline & Mean & SD & Mean & SD & Mean & SD \\
\hline \multicolumn{7}{|l|}{ Reading Interval } \\
\hline Preoperative & 8.9 & 4.8 & - & - & - & 1 \\
\hline 6 wk & 12.9 & 5.4 & 5.22 & 3.7 & - & - \\
\hline $3 \mathrm{mo}$ & 12.7 & 4.6 & 5.53 & 3.5 & 0.01 & 1.65 \\
\hline $6 \mathrm{mo}$ & 13.2 & 5.1 & 4.89 & 3.7 & 0.53 & 1.58 \\
\hline $1 \mathrm{yr}$ & 11.1 & 4.9 & 3.46 & 3.2 & 1.07 & 1.85 \\
\hline $2 \mathrm{yr}$ & 12.8 & 5.1 & 3.09 & 3.3 & 1.67 & 1.71 \\
\hline Final & 12.6 & 5.1 & 4.06 & 4.1 & 1.23 & 1.92 \\
\hline
\end{tabular}

ILA, intervertebral lordotic angle; SD, standard deviation.

tal views and 3.2 and $81.9 \%$ for coronal views. Twentytwo patients (34 levels) underwent repeat CT scan. For cases that had both a first and second CT, the means for number of bony bridges and percent fill seen on the initial CT were 3.6 and $83.8 \%$ on sagittal views and 3.2 and $79.4 \%$ on coronal views. The number of bony bridges counted on the sagittal views of the second CT increased $(p=0.01)$ by a mean of 0.38 to 4.0 from the first CT. The number of bony bridges counted on the second CT coronal views increased ( $p=0.006)$ by a mean of 0.47 to 3.7 from the first CT. Percent fill on the second CT sagittal views increased $(p=0.01)$ by a mean of $7.4 \%$ to $91.2 \%$ from the first CT. Coronal views from the second CT also showed an increase in percent fill $(p=0.003)$ by a mean of $9.6 \%$ to $89.0 \%$ from the first CT.

Radiographic outcomes are presented in Table 1. The modified Bridwell fusion grade tended to underrate the fusion status, as compared to CT scan, an accepted superior imaging modality for confirming fusion. At six months, fusion of at least one level (Bridwell grade 1) was assessed in 53\% patients. At one-year and final radiographic follow-up, fusion was assessed in $75 \%$ patients, as compared to $100 \%$ by CT criteria.

Preoperative mean ILA was $8.9^{\circ}$ (range, $0^{\circ}-20^{\circ}$; $\mathrm{SD}=4.8$ ); $12.9^{\circ}$ (range, $1^{\circ}-25^{\circ}$; $\mathrm{SD}=5.4$ ) at 6 weeks postoperatively; $12.7^{\circ}$ (range, $1^{\circ}-25^{\circ}$; $\mathrm{SD}=4.6$ ) at 3 months; $13.2^{\circ}$ (range, $0^{\circ}-27^{\circ} ; \mathrm{SD}=5.1$ ) at 6 months; and $11.1^{\circ}$ (range, $0^{\circ}-22^{\circ}$; $\mathrm{SD}=4.9$ ) at one-year. Postoperative ILA at six-weeks after surgery significantly increased by a mean of $3.94^{\circ}$ ( $\mathrm{SE}=0.64 ; 95 \% \mathrm{CI}=2.67,5.22$ ) over baseline; and this significant increase in ILA persisted through the time of final follow-up (mean final increase was $3.70^{\circ}$ ) $(\mathrm{SE}=0.67 ; 95 \% \mathrm{CI}=2.37,5.01)$. Mean lumbar lordosis was $37.7^{\circ}$ preoperatively (range, $1-67 ; \mathrm{SD}=13.6$ ) and increased $(p=0.06)$ by a mean of $2.85^{\circ}(\mathrm{SE}=1.48 ; 95 \% \mathrm{CI}=-0.14$, 5.84 ) to a mean of $40.6^{\circ}$ (range, $4^{\circ}-61^{\circ} ; \mathrm{SD}=11.9$ ) at final follow-up. Disc height significantly increased from baseline (average baseline disc height $=10.27 \mathrm{~mm} ; \mathrm{SD}=3.2$ ) to 6-weeks postoperative (average gain $=5.22 \mathrm{~mm} ; \mathrm{SD}=3.7$; $\mathrm{p}<0.001$ ); and this significantly increased height gain persisted through final follow-up (average gain $=4.06 \mathrm{~mm}$; $\mathrm{SD}=4.0 ; \mathrm{p}<0.001)$. Five patients ( $12 \%)$ had subsidence $\geq 4$ $\mathrm{mm}$, while $37 \%$ had subsidence $\geq 2 \mathrm{~mm}$.

Interobserver reliability varied depending on the measure tested. The ICC for ILA measures was 0.7 ( $\mathrm{SE}=0.09$; $95 \% \mathrm{CI}=0.52,0.88$ ), indicating good overall reliability between observers. The ICC for the total A and B measures was 0.89 ( $\mathrm{SE}=0.03 ; 95 \% \mathrm{CI}=0.83,0.95)$, showing excellent reliability between observers on these measures. For the number of bony bridges observed on CT, agreement had poor reliability with a kappa statistic of $0.10(\mathrm{SE}=0.06$; $\mathrm{z}=1.65 ; p=0.05)$ and an expected and actual agreement of $27.5 \%$ and $34.7 \%$, respectively. The same was true of percent fill, with a kappa statistic of 0.18 ( $\mathrm{SE}=0.09 ; \mathrm{z}=1.95$; $p=0.03$ ), expected agreement of $50.7 \%$ and actual agreement of $61.1 \%$. Bridwell fusion scores showed a slightly better agreement, but still poor reliability with a kappa of $0.38(\mathrm{SE}=0.09 ; \mathrm{z}=4.05 ; p<0.001)$.

\section{Complications}

Major complications were defined as any unplanned 
reoperation, and occurred in one patient. In this case, a 28-year-old non-smoking healthy male had persistent back pain six months after the index procedure (ALIF+anterior plate) due to failure of fusion and progressive subsidence of the interbody device. Re-operation consisted of posterior instrumented fusion, and solid fusion was attained. There were no intraoperative vessel injuries or major approach-related complications recorded in the medical records for this patient population.

Seven minor complications were identified: five cases with subsidence greater than $4 \mathrm{~mm}$; and two with device malposition. Subsidence $(\geq 4 \mathrm{~mm})$ was initially noted at follow-up visits from three months to two years after surgery in these five patients. Subsidence occurred in 2 of 9 levels $(22 \%)$ with a significant indentation of the endplate ('notching'), compared to $4 \%$ of levels without notching $(p=0.11)$. Each of the six cases of subsidence ultimately went on to fusion without revision by the final follow-up visit. In two cases, final device position was $4 \mathrm{~mm}$ anterior. Both levels fused without incident.

\section{Discussion}

A high rate of fusion was observed in this study which is similar to the rates reported in recent studies [4-8]. In addition the low rate of subsidence observed in this cohort ( $12 \%$ had $\geq 4 \mathrm{~mm} ; 37 \%$ had $\geq 2 \mathrm{~mm}$ ) compares favorably with historic results, which have been reported to be as high as $77 \%$ with other cages $[2,5,6]$. When subsidence did occur, it was often associated with superior endplate notching (22\% of the time), which suggests that surgical technique plays a role in the development of this phenomena [6]. This is also consistent with results in vitro demonstrating a significant decrease in compressive strength when the endplate is decorticated [12].

The modular device contains metallic spikes on its endplates that confer initial stability and may contribute to the high rate of fusion observed in this case series, even in the setting of stand-alone implantation (100\% fused). In a cadaveric study comparing this device to a stand alone to a polyehter ether ketone spacer, a dual tapered threaded cage and an intact spine, Buttermann et al. [13] found superior resistance to motion in flexion-extension, torsion and lateral bending with this device. The authors also demonstrated further decrease in motion with addition of an anterior plate or pedicle screw fixation [13]. Despite evidence of increased stability with supplemental instrumentation, in this case series no significant differences were detected in fusion rates between stand-alone or augmented cases. In fact the single reoperation for nonunion was in a case with supplemental anterior plate fixation. Although no cases of implant extrusion occurred in this cohort, further clinical experience with this device has suggested that posterior instrumentation may be indicated when considering ALIF in the setting of previous laminectomy. Regular postoperative radiographs are also recommended as important surveillance for implant extrusion.

The theoretical advantage of this device lies in its modularity, which allows configuration to provide maximum endplate coverage and restoration of intervertebral height. Previous authors have shown that engaging the peripheral vertebral rim and increasing the cage-implant contact area provides enhanced resistance to subsidence $[10,11,14]$. The ability to adjust disc-space distraction intraoperatively allows restoration of foraminal height and area that more closely reapproximates anatomic norms. The surgeon can directly increase the neuroforaminal area to varying degrees based on the size of the vertical struts implanted [15]. In addition to being rather resistant to subsidence, this ALIF technique allowed for significant increases in ILA and disc height that were maintained over the duration of follow-up.

Limitations of this study include those inherent in retrospective case series. The cohort included patients with a variety of diagnoses and surgical indications, including revision cases. There was variability in the use of supplemental fixation, which consisted of anterior or posterior instrumentation, although this did not appear to affect fusion status. All patients received bone morphogenic protein as a bone graft substitute, which has high-reported rates of fusion $[16,17]$; and therefore these results may not be reproducible if an alternative bone graft is used. The duration and type of radiographic follow-up were variable, although it should be noted that all patients received serial radiographic follow-up until fusion was observed. The high fusion rate and low number of patients precluded analyses of possible confounding factors such as age, smoking status and use of supplemental fixation. The retrospective nature of this study also diminished the likelihood of capturing all complications, especially minor ones, like ileus, or self-resolved ones, like transient radiculitis or retrograde ejaculation. Only complications specifically mentioned as such in postoperative clinic 
notes were included in our results. Additionally the lower two lumbar segments, L4/5 and L5/S1, represented a large majority of the fused levels within this population. While somewhat limiting our ability to determine the efficacy of this implant in the more proximal lumbar levels, this patient sample likely reflects the most common clinical scenario, as this technique for anterior interbody fusion is limited to the lumbar spine and the caudal lumbar segments are most frequently effected by degenerative change. Lastly a major limitation is the absence of validated self-reported patient outcome measures, especially when considering that fusion rate has not been shown to correlate with clinical outcome.

\section{Conclusions}

The results of this study show that a modular metallic intervertebral device for ALIF is efficacious in the promotion of bony fusion and resistance of postoperative subsidence. Despite a cohort that included a significant proportion of smokers, multiple level fusions and standalone ALIF, a high rate of fusion and maintenance of increased ILA and disc height were attained as assessed by a comprehensive radiograph analysis. Radiographs underestimate the incidence of fusion when compared to CT scans. Quantitative radiographic measures were the only ones to demonstrate good-excellent interobserver reliability.

\section{Conflict of Interest}

No potential conflict of interest relevant to this article was reported.

\section{References}

1. Kumar A, Kozak JA, Doherty BJ, Dickson JH. Interspace distraction and graft subsidence after anterior lumbar fusion with femoral strut allograft. Spine (Phila Pa 1976) 1993;18:2393-400.

2. McAfee PC. Interbody fusion cages in reconstructive operations on the spine. J Bone Joint Surg Am 1999;81:859-80.

3. Stauffer RN, Coventry MB. Anterior interbody lumbar spine fusion. Analysis of Mayo Clinic series. J Bone Joint Surg Am 1972;54:756-68.

4. Burkus JK, Sandhu HS, Gornet MF, Longley MC. Use of rhBMP-2 in combination with structural cortical allografts: clinical and radiographic outcomes in anterior lumbar spinal surgery. J Bone Joint Surg Am 2005;87:1205-12.

5. Cheung KM, Zhang YG, Lu DS, Luk KD, Leong JC. Reduction of disc space distraction after anterior lumbar interbody fusion with autologous iliac crest graft. Spine (Phila Pa 1976) 2003;28:1385-9.

6. Choi JY, Sung KH. Subsidence after anterior lumbar interbody fusion using paired stand-alone rectangular cages. Eur Spine J 2006;15:16-22.

7. Pavlov PW, Meijers H, van Limbeek J, et al. Good outcome and restoration of lordosis after anterior lumbar interbody fusion with additional posterior fixation. Spine (Phila Pa 1976) 2004;29:1893-9.

8. Sasso RC, Kitchel SH, Dawson EG. A prospective, randomized controlled clinical trial of anterior lumbar interbody fusion using a titanium cylindrical threaded fusion device. Spine (Phila Pa 1976) 2004;29:113-22.

9. Pfeiffer M, Griss P, Haake M, Kienapfel H, Billion M. Standardized evaluation of long-term results after anterior lumbar interbody fusion. Eur Spine J 1996;5:299-307.

10. Grant JP, Oxland TR, Dvorak MF. Mapping the structural properties of the lumbosacral vertebral endplates. Spine (Phila Pa 1976) 2001;26:889-96.

11. Closkey RF, Parsons JR, Lee CK, Blacksin MF, Zimmerman MC. Mechanics of interbody spinal fusion. Analysis of critical bone graft area. Spine (Phila Pa 1976) 1993;18:1011-5.

12. Oxland TR, Grant JP, Dvorak MF, Fisher CG. Effects of endplate removal on the structural properties of the lower lumbar vertebral bodies. Spine (Phila $\mathrm{Pa}$ 1976) 2003;28:771-7.

13. Buttermann GR, Beaubien BP, Freeman AL, Stoll JE, Chappuis JL. Interbody device endplate engagement effects on motion segment biomechanics. Spine J 2009;9:564-73.

14. Steffen T, Tsantrizos A, Aebi M. Effect of implant design and endplate preparation on the compressive strength of interbody fusion constructs. Spine (Phila Pa 1976) 2000;25:1077-84.

15. Wang M, Dalal S, Bagaria VB, McGrady LM, Rao $\mathrm{RD}$. Changes in the lumbar foramen following anterior interbody fusion with tapered or cylindrical cages. Spine J 2007;7:563-9. 
16. Burkus JK, Gornet MF, Schuler TC, Kleeman TJ, Zdeblick TA. Six-year outcomes of anterior lumbar interbody arthrodesis with use of interbody fusion cages and recombinant human bone morphogenetic protein-2. J Bone Joint Surg Am 2009;91:1181-9.
17. Burkus JK, Transfeldt EE, Kitchel SH, Watkins RG, Balderston RA. Clinical and radiographic outcomes of anterior lumbar interbody fusion using recombinant human bone morphogenetic protein-2. Spine (Phila Pa 1976) 2002;27:2396-408. 